Service social

\title{
Reflet d'une pratique psychosociale en oncologie
}

\section{Louise Picard}

Volume 45, numéro 3, 1996

\section{Santé}

URI : https://id.erudit.org/iderudit/706739ar

DOI : https://doi.org/10.7202/706739ar

Aller au sommaire du numéro

Éditeur(s)

École de service social de l'Université Laval

ISSN

1708-1734 (numérique)

Découvrir la revue

Citer cet article

Picard, L. (1996). Reflet d'une pratique psychosociale en oncologie. Service social, 45(3), 91-102. https://doi.org/10.7202/706739ar

\section{Résumé de l'article}

Le présent article traduit certains aspects de la pratique d'une travailleuse sociale œuvrant auprès de personnes atteintes de cancer et de leur famille. Cette pratique se vit à l'intérieur d'un centre hospitalier universitaire spécialisé en oncologie et se situe dans le contexte de la phase curative, c'est-à-dire du diagnostic jusqu'à la phase palliative. Sont présentés les modèles théoriques de référence ainsi que l'intervention psychosociale privilégiée. Le choix de l'intervention repose sur une expérience de pratique d'une dizaine d'années auprès de personnes atteintes de cancer, de leur famille et de leurs proches. 


\section{Reflet d'une pratique psychosociale en oncologie}

Louise PICARD

Travailleuse sociale, clinicienne-chercheure Centre hospitalier universitaire de Québec, Pavillon Hôtel-Dieu de Québec

L'apparition d'un cancer est une expérience de vie significative pour la personne atteinte. Celle-ci est brusquement envahie par un désarroi profond et elle doit affronter de multiples souffrances, qui prennent différentes formes : la douleur physique, rattachée à la présence des symptômes de la maladie, à l'action et aux conséquences des traitements; la douleur psychologique, liée à la perte de l'intégrité corporelle souvent perçue comme une trahison du corps, à l'incertitude de la guérison, à l'angoisse de la mort, à la cassure dans l'illusion de la toute-puissance et à la prise de conscience douloureuse de la finitude de la vie; la douleur sociale, associée aux remises en question et aux remises en jeu sur le plan des relations et des rôles familiaux et sociaux; la douleur existentielle, liée à une remise en question de sa raison de vivre, du sens de son existence et de la vie. 
Le cancer entraîne aussi un bouleversement chez les proches : le conjoint, les enfants, la famille et les amis. La menace de perdre une personne significative et aimée provoque chez eux une détresse psychologique et suscite des changements dans les rôles et responsabilités (Northouse, $1991 ;$ 1995). Ces personnes sont donc déstabilisées sur le plan personnel, conjugal et familial. Tous ces changements et bouleversements engendrent un fardeau supplémentaire et un risque élevé de détresse et d'épuisement (Issel, Ersek et Lewis, 1990 ; Lewis et Hammond, 1993). Chacun utilisera différentes stratégies pour dépasser cet «état de souffrance globale» et retrouver une paix et une sécurité intérieures.

Le cancer revêt un caractère menaçant parce qu'il force à affronter la mort et un caractère vital parce qu'il projette au cœur de la lutte pour la vie. Le travailleur social se retrouve donc devant une expérience humaine complexe et profonde qui souvent le dépasse et qui lui est, par sa complexité, difficile à comprendre et à intégrer. Mais cette recherche de compréhension reste fascinante.

Aux fins de cet article, nous abordons l'intervention dans le contexte de la phase curative, qui s'étend du diagnostic jusqu'à la phase palliative. Le texte se divise en deux parties. Nous présentons dans un premier temps les modèles théoriques de référence qui sous-tendent notre pratique et, dans un deuxième temps, le modèle d'intervention élaboré peu à peu au cours des dix dernières années d'expérience auprès de personnes atteintes de cancer et de leurs familles.

\section{LES MODÈLES THÉORIQUES DE RÉFÉRENCE DANS NOTRE PRATIQUE}

Différents modèles de compréhension circulent dans le milieu scientifique relativement aux notions de santé et de maladie. Trois modèles théoriques ont eu davantage d'influence sur notre pratique professionnelle: le modèle biomédical traditionnel, le modèle psychosomatique et le modèle psychoneuro-immunologique.

Le modèle biomédical est centré essentiellement sur la maladie. Celle-ci est définie comme une affection; on parle d'altération de l'état de santé se manifestant par des symptômes et des signes. Le traitement vise à éliminer les symptômes. La 
conception de la maladie fait donc référence ici à des réalités tangibles, mesurables et observables. C'est le domaine de l'explicite. L'annonce du diagnostic de cancer et la proposition d'un traitement ont pour fonction de préciser la nature de la maladie et les moyens concrets à prendre pour la traiter. Cette étape du diagnostic est aussi une source de bouleversement. C'est un moment crucial sur le plan psychosocial, car c'est souvent le point de départ de notre engagement comme travailleuse sociale. La personne malade et sa famille ont besoin que leur détresse soit écoutée et nommée pour les apaiser et susciter de l'espoir.

Le modèle psychosomatique issu de la psychanalyse s'attache à comprendre et à préciser les relations au corps, la place de la pensée et des émotions dans la dynamique et la structure de la personne. Ce courant a élargi la vision psychanalytique classique en cherchant à intégrer et à articuler les multiples facteurs en cause dans la maladie (génétiques, biologiques, psychologiques, sociaux et environnementaux). L'intérêt particulier de ce courant est qu'il propose une notion charnière dans la compréhension de cette articulation entre le corps et l'esprit. Il s'agit de l'état de désarroi associé à une «impasse relationnelle». Celle-ci est définie comme une situation déstabilisante ou menaçante (pertes vécues, conflit intrapsychique, interpersonnel, etc.) dans laquelle la personne est prisonnière et où la lutte ou la fuite se révèle impossible, inutile, voire perdue (Crombez, 1995). Dans cette vision, l'impasse se construit à partir, d'une part, de la perception subjective de l'événement, de la signification que la personne lui donne et, d'autre part, de l'évaluation de ses capacités psychiques à appréhender et à élaborer des solutions en vue de résoudre cette impasse. Le maintien prolongé de l'impasse ancre la personne dans un état de désarroi; elle se sent piégée, sa capacité de réagir diminue, une désorganisation physique et psychique s'installe et crée un terrain prédisposant et propice à l'apparition de la maladie (Crombez, 1995). Cependant, contrairement à ce qui est véhiculé, il ne saurait être question de responsabilité, parce que cette impasse s'est construite à un moment de détresse, d'urgence et de menace où la personne n'était pas en mesure de choisir délibérément cette issue (Crombez, 1995 ; Gauthier, 1995 ; Lefebvre, 1980 et 1984; Leroux, 1992).

La psychanalyse et la psychosomatique introduisent une dimension subjective susceptible de participer à la psychogenèse de la maladie. Dans cette perspective, le cadre thérapeutique vise à offrir un lieu où la souffrance peut se dire et s'élaborer de façon 
telle qu'un sens peut surgir. La subjectivité de la personne est au centre du processus.

Enfin, les théories du stress inspirées de travaux de physiologistes (Cannon, 1929) permettent de mieux comprendre les mécanismes neuro-humoraux en jeu dans l'interaction entre le stress et le système immunitaire (Boucher et Binette, 1981). Elles ont établi une relation temporelle entre les stresseurs et l'occurrence de la maladie. La diminution de la résistance de l'organisme sous l'influence de stresseurs psychosociaux a pour effet de vulnérabiliser la personne sur le plan biologique et d'accroître les risques de l'apparition d'une maladie. Ces théories du stress ont ouvert la voie à la psychoneuro-immunologie. Cette discipline s'intéresse aussi à l'étude des processus biologiques liant le stress psychologique et l'immunité. Elle apporte un éclairage complémentaire en identifiant les facteurs psychologiques et sociaux qui influencent la diminution de la résistance des individus à la maladie. Des travaux ont permis d'expliquer les relations complexes entre un état de stress prolongé, l'affaiblissement du système immunitaire et les risques d'apparition de la maladie (Bovbjerg, 1989; Fillion, 1993).

Les études de Lazarus et Folkman (1984) en psychologie cognitive ont dégagé certains déterminants de l'état de stress, à savoir les facteurs situationnels, les facteurs personnels, les stratégies de coping et l'évaluation cognitive des stresseurs. Pour ceux-ci, l'étude du stress psychologique porte sur le processus d'interaction individu-environnement. Le stress est perçu comme la résultante de cette interaction (Fillion, 1993). Des recherches liées plus spécifiquement au cancer ont relevé des variables psychosociales associées à une meilleure adaptation. Ainsi, l'évaluation cognitive des conséquences associées à un cancer, les types de stratégies d'adaptation utilisées et le sentiment de contrôle sur la maladie jouent un rôle sur le processus d'adaptation de la personne (Fillion, 1993). Un meilleur sentiment de maîtrise de la situation, une perception cohérente de la maladie, des stratégies d'adaptation efficaces favorisent un meilleur fonctionnement du système immunitaire qui, à son tour, augmente les probabilités de survie. De même, la croyance en la guérison, le désir de vivre et une démarche personnelle sont des facteurs pouvant contribuer à l'amélioration du système immunitaire, donc du pronostic de vie ou du moins de la qualité de vie (Fillion, 1993).

Globalement, ces modèles théoriques de compréhension font ressortir la multidimensionnalité des mécanismes et des 
facteurs liés à la santé et à la maladie. La médecine se centre sur les aspects physiques; la psychanalyse sur sa dimension subjective; la psychosomatique et la psychoneuro-immunologie sur les liens entre le corps et l'esprit. Ces modèles reconnaissent tous, à des degrés divers et par une démarche différente, non seulement le rôle joué par les processus physiques, mais aussi l'influence des facteurs cognitifs, psychologiques, relationnels et environnementaux pour la reprise des fonctions (psychique, immunitaire, d'adaptation, etc.) susceptibles d'aider au rétablissement ou au maintien de la santé. Il n'existe pas de contradiction fondamentale sur cette question, les nuances et les distinctions s'exprimant sur le plan de l'épistémologie et de la nature de l'intervention.

Notre philosophie d'intervention repose donc sur la conception que la santé englobe tous ces facteurs bio-psychosociaux. En effet, tout n'est pas physique ou psychologique, et surtout rien n'est magique. La réalité est beaucoup plus complexe. Notre pratique tient compte de ces facteurs, parce qu'ils rejoignent les différentes manifestations de la souffrance vécue par les personnes atteintes de cancer ainsi que leurs préoccupations telles qu'elles s'expriment dans leur discours. Cette compréhension nous a permis de donner une assise théorique et une direction à notre intervention. Il s'agit d'une démarche particulièrement importante en milieu hospitalier en raison de tout ce qui est véhiculé en matière de tendances et de croyances par les représentants des différentes disciplines du milieu et par la clientèle.

\section{L'INTERVENTION PSYCHOSOCIALE AUPRÈS DE LA PERSONNE ATTEINTE DE CANCER}

\section{Phase I: I'état de crise chez la personne atteinte de cancer}

Comment se présente la personne atteinte de cancer? Cette personne vient d'apprendre qu'elle a un diagnostic de cancer, une récidive ou qu'elle a atteint la phase palliative de sa maladie. Elle est en état de choc. Elle est envahie par ce qui lui arrive: ses symptômes, ses douleurs physiques, son diagnostic, ses traitements et l'incertitude quant à son avenir. Va-t-elle vivre, survivre ou mourir? Et comment? Aura-t-elle les ressources personnelles pour traverser cette période douloureuse de sa vie et composer avec la menace de la mort? 
Elle est submergée par une multitude d'émotions, surtout la peur de mourir, de rester diminuée, impotente, de souffrir et de faire souffrir ceux qu'elle aime. Les doutes l'assaillent et la déchirent. Les moments d'espoir et de certitude qu'elle va guérir alternent avec des moments d'incertitude et de désarroi face à son avenir. Sa vie vient de chavirer. Son univers est réduit à son présent et à son corps souffrant. Elle est en état de déséquilibre: c'est l'état de crise. L'angoisse exprimée alors est celle provoquée par son corps malade. C'est cette angoisse qui lui est - et nous est - d'abord la plus accessible. La personne se sent fragile et vulnérable, elle a perdu ses moyens habituels de fonctionner et, pourtant, elle doit faire des choix cruciaux pour sa vie.

Trois enjeux se présentent pour elle à cette étape : l'intégration des émotions liées à l'impact de la maladie, le choix de lutter pour sa vie et la reprise d'un fonctionnement psychoaffectif et social adapté.

\section{Premier enjeu: I'intégration des émotions}

liées à l'impact de la maladie

Les émotions ressenties par la personne atteinte de cancer sont intenses, multiples et souvent même contradictoires. Le premier besoin exprimé est qu'on l'aide à réduire la tension et le degré d'anxiété à un niveau supportable, afin qu'elle puisse gérer les principaux éléments de la situation. Elle a besoin de sentir qu'elle garde un pouvoir sur sa vie. Son défi consiste à rester en contact avec ce qu'elle vit à l'intérieur d'elle-même, tout en reprenant la maîtrise de ce tumulte émotionnel. La sauvegarde du lien avec sa vie intérieure l'aidera dans les nombreuses décisions qu'elle aura à prendre, de même que pour la reprise de son équilibre et son adaptation à la situation.

\section{Deuxième enjeu: le choix de lutter pour sa vie}

Ce choix s'élabore et s'actualise à travers les décisions de traitements. L'urgence de la décision et le manque de temps entrent en contradiction avec la nature grave, profonde et lente de la réflexion nécessaire. Car la décision d'accepter les traitements doit s'appuyer aussi sur un désir et une volonté bien arrêtés de vivre. La réflexion liée à cette décision se pose à la phase initiale du diagnostic et elle est susceptible de se poser de nouveau au cours de la maladie, puis à chaque événement inquiétant ou résultat décevant. Ces phases décisionnelles sont particulièrement cruciales. Elles peuvent être une source de souffrance si les 
chances de guérison sont limitées ou ne sont pas celles qu'espère la personne. Les questions qui hantent celle-ci et auxquelles elle doit répondre sont les suivantes: pourquoi se battre? est-ce que cela en vaut la peine? Les réponses ne viennent pas nécessairement d'emblée.

Troisième enjeu: la reprise $d^{\prime}$ un fonctionnement psychoaffectif et social adapté

Après cette vague de fond, la personne découvre parfois avec étonnement ou avec soulagement que la vie continue. Elle mobilise ses ressources personnelles pour entamer la période de traitements et pour poursuivre sa vie en tenant compte des changements survenus. Cependant, les ajustements sont nombreux, les points de repère habituels ont disparu. Les choses ne sont plus perçues et vécues de la même façon. Les priorités de vie sont à repenser.

Dans ce mouvement, la personne malade utilisera différentes stratégies pour reprendre son équilibre et s'adapter à sa nouvelle réalité. Cette adaptation se réalisera à l'intérieur d'un processus où elle apprendra à apprécier et à gérer les nouvelles exigences liées à la maladie, à maintenir son estime de soi et son équilibre émotionnel, tout en conservant des relations satisfaisantes avec les autres.

\section{Les objectifs et les stratégies d'intervention}

À cette étape, c'est l'intervention en situation de crise. Elle vise dans un premier temps à réduire à un niveau supportable la tension et l'anxiété provoquées par l'impact de la maladie. Elle vise à rétablir l'équilibre de la personne en mobilisant ses ressources personnelles, en favorisant la restructuration cognitive de la situation, la reprise d'un espoir réaliste et la maîtrise sur l'événement et sur certains aspects de sa vie (réappropriation). Dans un second temps, l'intervention a pour objectif de soutenir la personne dans son processus d'adaptation.

Les stratégies d'intervention utilisées sont: l'expression des émotions (peur, inquiétudes, incertitude, doutes, perte d'espoir, espoir, etc.) ; l'expression et le recadrage des différentes perceptions liées au cancer et aux traitements; l'information et la normalisation des différentes étapes de la crise; l'identification des façons habituelles de vivre ses expériences personnelles et la validation de leur efficacité ; l'apprentissage de nouvelles stratégies d'adaptation et une réflexion sur le désir de vivre, de se battre ainsi que sur les raisons de vivre. 


\section{Phase II: la quête de sens et la recherche de vie pour la personne atteinte}

Lorsque l'angoisse liée à la maladie est apaisée et que les émotions sont ramenées à un niveau supportable, la personne découvre que rien n'est plus pareil. Elle s'interroge sur sa façon d'être, d'entrer en relation avec elle-même et avec les autres. Sa compréhension d'elle-même, des autres et des événements de sa vie devient différente. Subitement, une réévaluation du rapport à soi, aux autres et à la vie s'amorce. Plusieurs personnes deviennent alors disposées à s'ouvrir à d'autres types de souffrances à l'intérieur d'elles-mêmes et à rechercher un sens à cette expérience apparemment insensée. Pourquoi ? Pourquoi moi?

Les premières réponses surgissent et des événements douloureux, passés et présents, remontent à la surface. Ces personnes commencent à parler de leur vie, de séparations, de pertes, de ruptures, de désillusions, de déceptions, en fin de compte des blessures ouvertes ou mal cicatrisées qui ont été souvent vécues dans la solitude et le silence. Des blessures de tout ordre (personnel, interpersonnel) qui n'ont pu être dites, écoutées et qui sont demeurées sans solution satisfaisante. La personne est toujours dans une impasse et elle poursuit sa quête d'une "solution de vie» pour s'en sortir. Cette solution passera fréquemment par une relecture de certains passages de sa vie.

Le principal enjeu: le choix d'une ouverture ou d'une fermeture à son expérience intérieure et à une recherche de sens et de réappropriation de sa vie

Devant l'émergence du passé et de ce présent trop lourd la personne se retrouve à la croisée des chemins. Plusieurs n'iront pas plus loin et tenteront de reprendre leur vie telle qu'elle était avant d'être interrompue par l'avènement de la maladie. La peur d'être submergés par leur vécu émotionnel les fait se sentir vulnérables et les amène à se refermer. Par contre, d'autres exprimeront le désir d'aller plus loin, parce que la maladie les force à se pencher sur le sens de leur souffrance et de leur existence. Ils choisissent alors de faire de cette expérience douloureuse une occasion de grandir, de changer en mieux.

\section{Les objectifs et les stratégies d'intervention}

À cette étape, l'intervention vise à favoriser l'expression et l'identification des sources de souffrance. Elle vise l'éveil et le 
renforcement des forces vitales et la découverte de la créativité et de l'originalité de la personne (blessures, vulnérabilité, forces, créativité, solutions, etc.). Elle guide et elle soutient la recherche du sens et des raisons de vivre.

La stratégie principale d'intervention consiste à fournir un lieu et un cadre relationnel propices à l'expression de la souffrance et à l'élaboration de solutions personnelles. Ce cadre thérapeutique se présente comme un moment et un espace favorables à la découverte de la trame de vie et propices à l'éveil de la vitalité (forces vitales) enfouie.

\section{L'INTERVENTION AUPRÈS DE LA FAMILLE ET DES PROCHES}

L'annonce du cancer crée aussi une détresse psychologique chez les proches: le conjoint, les enfants et la famille élargie. La menace de perdre un être significatif leur fait prendre conscience de leur propre mort et de l'incertitude de leur avenir. Cette menace permet de mesurer l'attachement à cette personne et l'importance de la place que celle-ci occupe dans leur vie. En même temps, elle les met brutalement en contact avec la «finitude» du lien, la possibilité de la perte et de la séparation. Leur sollicitude et leur attachement les amènent à se demander quelle attitude adopter. Comment aider à soulager la souffrance? Doivent-ils parler ouvertement de ce qu'ils vivent ou se taire pour protéger l'autre? etc.

Dans un autre registre, les nombreuses demandes liées à la maladie ont pour conséquence d'amener des changements et une réaffectation dans les rôles et dans les responsabilités familiales. Tous les membres de la famille vivent donc une période de crise et d'adaptation.

Trois enjeux se présentent: la protection ou l'établissement du soutien mutuel au sein du couple et de la famille, la sauvegarde de la cohésion du couple et de la famille, de même que le maintien de l'investissement des ressources familiales et la prévention de leur épuisement.

Premier enjeu: la protection ou l'établissement du soutien mutuel au sein du couple et de la famille

Le conjoint et les membres de la famille sont les personnes les plus près de la personne atteinte. En raison de cette proximité ils jouent un rôle important de soutien. Toutefois, puisqu'ils sont 
eux-mêmes bouleversés par l'événement, ils ont besoin d'être soutenus pour pouvoir remplir adéquatement ce rôle. C'est particulièrement vrai lorsque la famille touchée comporte de jeunes enfants. Il est bien reconnu maintenant que le soutien social (Bozzini et Tessier, 1985) est un facteur de protection dans le processus d'adaptation et le rétablissement de la santé psychosociale de la famille.

Deuxième enjeu: la sauvegarde de la cohésion du couple et de la famille

On réalisera cette cohésion en assurant une continuité dans le respect des besoins et des activités individuelles de chaque membre de la famille et en maintenant une continuité dans l'investissement du lien conjugal et de la vie familiale. Le soutien mutuel et la cohésion sont des facteurs déterminants pour la reprise et le maintien de l'équilibre du couple et de la famille.

Troisième enjeu: le maintien de l'investissement des ressources familiales et la prévention de l'épuisement des ressources familiales

Les nombreuses exigences inhérentes à la maladie et à son traitement, liées à la diminution des capacités de la personne malade, risquent de mobiliser considérablement chaque membre de la famille en lui imposant une charge additionnelle. Le recours à des ressources communautaires pour éviter un épuisement ou un désinvestissement prématuré doit parfois être envisagé.

\section{Les objectifs et les stratégies d'intervention}

Notre intervention auprès des proches vise donc à favoriser le soutien mutuel par l'apprentissage d'une communication ouverte et d'une compréhension réciproque des réactions. Elle vise à renforcer le lien conjugal et les liens affectifs familiaux, tout en favorisant la poursuite et le respect de la vie individuelle. Elle vise aussi à réduire le fardeau de chacun et à prévenir l'épuisement de la famille en introduisant l'utilisation de ressources communautaires complémentaires.

Les stratégies d'intervention utilisées sont : l'information et la normalisation des différents éléments et étapes de la crise; l'identification de leurs stratégies d'adaptation, la validation de leur efficacité ; l'apprentissage de stratégies d'adaptation liées à 
une communication ouverte et à une compréhension mutuelle des besoins et des réactions et, enfin, l'utilisation de ressources de la communauté (CLSC, organismes d'entraide, etc.).

\section{CONCLUSION}

Notre intervention auprès des personnes atteintes de cancer et de leurs proches comporte plusieurs volets, afin que soient prises en considération les différentes formes de souffrances vécues. Le but ultime est d'amener la personne atteinte de cancer à identifier et à trouver des solutions satisfaisantes aux difficultés qu'elle affronte. Elle aura ainsi l'occasion d'accroitre le pouvoir $q u$ 'elle a sur sa vie, celui-ci étant intensifié par une plus grande connaissance d'elle-même. Notre intervention consiste à éveiller la personne à une relecture de sa vie, l'amenant ainsi à envisager et à espérer de nouveaux moyens plus appropriés pour faire face à sa réalité et augmenter ses forces vitales. Cependant, la condition préalable est que la personne exprime le désir d'amorcer une telle démarche de changements et décide d'accomplir ellemême cette transformation. Il ne peut y avoir de croissance sans l'espoir et la volonté d'aller vers quelque chose de meilleur. Mais, quelle que soit la décision prise par la personne, nous nous devons de la respecter dans son choix et dans son rythme de progression, parce que cette personne sait au fond d'elle-même ce qu'il y a de mieux pour elle. Aller au-delà de cette décision serait la projeter dans une situation plus intolérable encore.

Notre intervention reconnaît aussi les répercussions de la maladie sur le réseau affectif, de même que les interinfluences de l'individu et de son environnement. Elle veut s'assurer que les conditions optimales de développement et d'adaptation sont présentes et se poursuivent tant pour la personne atteinte de cancer que pour son réseau naturel de soutien. La réalisation de ces objectifs peut nécessiter une intervention auprès du couple, de la famille, une intervention individualisée auprès d'un proche (conjoint, enfants, etc.) et une liaison avec des organismes communautaires.

Cependant, malgré l'existence de nombreux travaux de recherche dans le domaine des aspects psychosociaux du cancer et d'une importante littérature sur le sujet, la complexité et la richesse de cette expérience humaine restent à comprendre et à découvrir. 


\section{Références bibliographiques}

BOUCHER, F., et A. BINETTE (1981). Bien vivre le stress, Bourcherville: Éditions de Mortagne.

BovbJerg, D. (1989). "Psychoneuroimmunology and cancer», dans J.C. Holland et H. Rowland, Handbook of Psychooncology, New York, Oxford University Press, p. 727-734.

BozZINI, L., et R. TESSIER (1985). "Support social et santé», dans Jacques Dufresne, Fernand Dumont, Yves Martin, Traité d'anthropologie médicale. L'Institution de la santé et de la maladie, Québec: Presses de l'Université du Québec, Institut québécois de recherche sur la culture; Lyon: Presses universitaires de Lyon, p. 905-939.

CANNON, W.B. (1929). Bodily Changes in Pain, Hunger, Fear and Rage: An Account of Recent Researches into the Function of Emotional Excitement, New York: Appleton.

Crombez, J.C. (1994). La guérison en écho, Beauport: Publications MNH.

FILLION, L. (1993). Évaluation cognitive, état de stress et immunité lors d'un diagnostic de tumeur au sein. Thèse de doctorat. Bibliothèque générale, Université Laval.

GAUTHiER, J. (1994). "Corps en souffrance et quête de sens », dans Le processus de guérison par-delà la souffrance ou la mort, Beauport: Publications MNH, p. 43-48.

ISSEL, L.M., M. ERSEK et F.M. LEWIS (1990). «How children cope with mother's breast cancer», ONF, vol. 17, $\mathrm{n}^{\circ} 3$, p. 5-12.

LAZARUS, R.S., et S. FOLKMAN (1984). "Coping and adaptation », dans Handbook of Behavioral Medicine, New York: The Guilford Press, p. 283-324.

LEFEBVRE, P. (1980). «The narcissistic as a determinant of psychosomatic disorder ", The Psychiatric Journal of the University of Ottawa, vol. V, $\mathrm{n}^{\mathrm{o}} 1$, mars, p. 5-11.

LEFEBVRE, P. (1984). «La psyché de la somatose en quête de névrose: le sentier obstrué », Revue française de psychanalyse, vol. 5, p. 11731181.

LEROUX, J.R. (1992). Le malade somatisant: un corps douloureux en manque de pensées, Colloque Wilfred R. Bion, Société psychanalytique de Montréal, mars.

LEWIS, F.M., M.A. HAMMOND et M.F. Woods (1993). "The family's functioning with newly diagnoses breast cancer in the mother: the development of an explanatory model », Journal of Behavioral Medicine, vol. 16, $\mathrm{n}^{\circ} 4$, p. 351-370.

NORThOUSE, L.L. (1995). "The impact of cancer in women on the family », Cancer Practice, mai-juin, vol. 3, no 3, p. 134-142.

Northouse, L.L., et A. Cracchiol-Caraway (1991). "Psychologic consequences of breast cancer on partner and family», Seminars in Oncology Nursing, vol. 7, no 3, août, p. 216-223. 